\title{
HIGHER RANK NUMERICAL HULLS OF MATRICES
}

\author{
ABBAS SALEMI
}

Abstract. For any $n \times n$ matrix $A$, we use the joint higher rank numerical range, $\Lambda_{k}\left(A, \ldots, A^{m}\right)$, to define the higher rank numerical hull of $A$. We characterize the higher rank numerical hulls of Hermitian matrices. Also, the higher rank numerical hulls of unitary matrices are studied.

Mathematics subject classification (2010): 15A60,81P68.

Keywords and phrases: Higher rank numerical range, polynomial numerical hull, quantum computing, higher rank numerical hull.

\section{REFERENCES}

[1] Man-Duen Choi, DaVid W. KRIBS, AND KAROL Ż YCZKOWSKI, Quantum error correcting codes from the compression formalism, Rep. Math. Phys. 58 (2006), 77-91.

[2] Man-Duen Choi, John A. Holbrook, David W. Kribs, and Karol Ż YCZKows Ki, Higherrank Numerical Range of Unitary and Normal Matrices, Operators and Matrices 1, 3 (2007), 409-426.

[3] Chandler Davis AND ABBAS S ALEMI, On polynomial numerical hulls of normal matrices, Linear Algebra Appl. 383 (2004), 151-161.

[4] Chandler Davis, Chi-Kwong Li and Abbas Salemi, Polynomial Numerical Hulls of Matrices, Linear Algebra Appl. 428 (2008), 137-153

[5] AnNe GREenbaum, Generalizations of the field of values useful in the study of polynomial functions of a matrix, Linear Algebra Appl. 347 (2002), 233-249.

[6] Chi-Kwong Li, Yiu-Tung Poon and Nung-Sing Sze, Condition for the higher rank numerical range to be non-empty, Linear and Multilinear Algebra 57 (2009), 365-368.

[7] Chi-Kwong Li And Nung-Sing Sze, Canonical forms, higher rank numerical ranges, totally isotropic subspaces, and matrix equations, Proc. Amer. Math. Soc. 136 (2008), 3013-3023.

[8] Olavi Nevanlinna, Convergence of iterations for linear equation, Birkhäuser, Basel, 1993.

[9] Hugo J. Woerdeman, The higher rank numerical range is convex, Linear and Multilinear Algebra 56, 1-2 (2008), 65-67. 